\title{
Moderate Dysplasia
}

National Cancer Institute

\section{Source}

National Cancer Institute. Moderate Dysplasia. NCI Thesaurus. Code C8363.

A morphologic finding indicating the presence of moderate cellular atypia associated with moderate architectural changes in a tissue sample. 